Kristina van Deuverden*

\title{
Nur geringer haushaltspolitischer Spielraum trotz hoher Überschüsse
}

DOI 10.1515/zfwp-2017-0004

\begin{abstract}
Public budgets in Germany developed favorable during the last years. Currently there is a broad discussion on how to spend unexpected budget surpluses: tax reductions and a various number of spending purposes are on the agenda. Many of the proposals overlook that existing surpluses partly reflect special developments and are, thus, only temporary. This part of surpluses indicates no scope for tax reductions or lasting expenditure, but it can temporarily be used on investment spending and, thus, can enhance potential growth. In many cases proposals also overlook that the development of public finances in Germany is very heterogeneous. Regarding demographic developments social security systems will face heavy challenges and will have to raise social contribution rates in near future. A medium-term projection indicates an increase of nearly four percentage points till 2025. This dampens economic development and reduces potential growth. Thus, the permanent part of the budget surplus should be used to increase tax transfers to social insurances - especially because a huge volume of non-insurance benefits is still financed within the security system.
\end{abstract}

\section{Einleitung}

Seit einigen Jahren entwickeln sich die öffentlichen Haushalte immer wieder besser als ursprünglich geplant. Dies gilt insbesondere für den Bundeshaushalt: Trotz milliardenschwerer Mehrausgaben wegen der hohen Flüchtlingsmigration konnte er im vergangenen Jahr wieder mit einem unerwarteten Überschuss abschließen. Auch die Länder insgesamt schrieben schwarze Zahlen. Für die Kommunen sieht es ähnlich aus und auch bei den Sozialversicherungen war die Finanzlage entspannt. So ist es - zumal in einem Wahljahr - kein Wunder, dass hitzig darüber diskutiert wird, wie diese Überschüsse verwendet werden sollen. Auf der einen Seite steht dabei die Forderung nach Steuersenkungen, auf der anderen die nach Ausgabeerhöhungen. Dazwischen steht der Finanzminister, der sowohl Mindereinnahmen wie auch Mehrausgaben möglichst begrenzen will und

*Kontakt: Kristina van Deuverden, DIW Berlin, Mohrenstraße 58, 10117 Berlin, Tel.: 030/ 89789466, E-Mail: kvandeuverden@diw.de 
darauf verweist, dass in der Finanzplanung des Bundes bereits für das kommende Jahr Lücken klaffen.

Bevor über die Verwendung der „hohen“ Überschüsse entschieden werden kann, sollten sie etwas näher beleuchtet werden. So sagt das Vorhandensein von Überschüssen allein noch wenig darüber aus, ob beziehungsweise welche Spielräume die Finanzpolitik hat. Dazu muss geklärt werden, ob die Überschüsse konjunkturell bedingt oder ob sie dauerhaft sind. Auch können sie durch Sonderfaktoren verursacht sein. Wichtig ist zudem, ob alle Haushalte gut aufgestellt sind oder ob eine Schieflage besteht, die sich in Zukunft - mit und ohne jetzt beschlossene Maßnahmen - verstärkt. Erst wenn geklärt ist, welche Spielräume wirklich bestehen, kann darüber diskutiert werden, wie sie verwendet werden sollen und welche Maßnahmen geeignet sind, das potentielle Wirtschaftswachstum zu erhöhen.

\section{Bestandsaufnahme: Deutliche Verbesserung der öffentlichen Finanzen in den vergangenen Jahren}

Nach der Jahrtausendwende verschlechterte die Lage der öffentlichen Haushalte sich rapide. Die im Maastrichter Vertrag vorgesehene Obergrenze für das Budgetdefizit wurde immer wieder gerissen und aus Brüssel hagelte es blaue Briefe. Dieses Bild hat sich in den vergangenen Jahren in sein Gegenteil verkehrt und die öffentlichen Haushalte entwickelten sich immer wieder besser als vorhergesehen. Dies lag allerdings nur in geringem Maße an der Finanzpolitik selbst, sondern vielmehr an der wirtschaftlichen Entwicklung und an den stetig sinkenden Zinsausgaben.

Die deutsche Wirtschaft konnte in den vergangenen Jahren merklich zulegen. Das Wachstum war dabei mehr und mehr von der Binnenwirtschaft getragen und ging mit einem kräftigen Beschäftigungsaufbau einher, der vorwiegend im sozialversicherungspflichtigen Bereich stattfand. Die Bruttolohnsumme stieg und die Arbeitsplatzsicherheit nahm zu. Dies hellte die Kauflaune der privaten Haushalte auf; privater Konsum und Wohnungsbau expandierten spürbar. Bei dieser wirtschaftlichen Entwicklung konnten sowohl die aufkommensstärksten Steuern, die Lohn- und die Mehrwertsteuer, wie auch die Einnahmen aus Sozialbeiträgen kräftig zulegen. Gleichzeitig gingen die Ausgaben für Arbeitslosengeld und Arbeitslosengeld II deutlich zurück. ${ }^{1}$

1 Die Ausgaben für Lohnersatzleistungen gehen seit Mitte des vergangenen Jahrzehnts stetig zurück, unterbrochen lediglich in den beiden, auf die Finanzkrise folgenden Jahren. Im vergangenen Jahr lagen die Ausgaben der Arbeitslosenversicherung für Arbeitslosen-, Kurzarbeiter-, Insolvenzgeld etc. sowie die Ausgaben des Bundes für Arbeitslosengeld II um etwa 21 Milliarden Euro 
Die öffentlichen Haushalte wurden zudem dadurch entlastet, dass die Ausgaben für den Schuldendienst merklich sanken. ${ }^{2}$ Dies lag nicht allein an dem allgemein niedrigen Zinsniveau. Der deutsche Fiskus konnte vor allem von einer starken Verunsicherung profitieren, die immer wieder neu aufflackerte. Auf Sicherheit bedachte Kapitalanleger waren bereit, Bundesanleihen zu für den deutschen Fiskus sehr günstigen Konditionen zu erwerben - zum Teil akzeptierten sie sogar negative Renditen.

Tabelle 1: Wichtige finanzwirtschaftliche Kennziffern in den Jahren 2005 bis $2015^{1}$

- in Relation zum nominalen Bruttoinlandsprodukt -

$\begin{array}{lllllllllll}2005 & 2006 & 2007 & 2008 & 2009 & 2010 & 2011 & 2012 & 2013 & 2014 & 2015\end{array}$

Nominaler

\begin{tabular}{llllllllllll} 
Finanzierungssaldo & $-31 / 2$ & $-13 / 4$ & $1 / 4$ & $-1 / 4$ & $-31 / 4$ & $-41 / 4$ & -1 & 0 & $-1 / 4$ & $1 / 4$ & $3 / 4$ \\
\hline
\end{tabular}

Struktureller

\begin{tabular}{lccccccccccc} 
Finanzierungssaldo $^{2}$ & -2 & $-13 / 4$ & -1 & $-11 / 4$ & $-3 / 4$ & $-31 / 2$ & $-11 / 2$ & $-1 / 4$ & 0 & $1 / 2$ & $3 / 4$ \\
\hline Primärsaldo & $-3 / 4$ & 1 & 3 & $21 / 2$ & $-1 / 2$ & -2 & $11 / 2$ & $21 / 4$ & $13 / 4$ & 2 & $21 / 4$
\end{tabular}

Struktureller

\begin{tabular}{llllllllllll} 
Primärsaldo & $3 / 4$ & 1 & $13 / 4$ & $11 / 2$ & 2 & -1 & 1 & 2 & 2 & $21 / 4$ & $21 / 4$ \\
\hline
\end{tabular}

\begin{tabular}{llllllllllll}
\hline Schuldenstandsquote $^{3}$ & 67 & $661 / 2$ & $633 / 4$ & 65 & $721 / 2$ & 81 & $783 / 4$ & 80 & $771 / 2$ & 75 & $71 \frac{1}{4}$ \\
\hline
\end{tabular}

${ }^{1}$ In Abgrenzung der Volkswirtschaftlichen Gesamtrechnungen. ${ }^{2}$ Berechnet nach dem für die deutsche Schuldenbremse geltenden Verfahren. ${ }^{3}$ In Abgrenzung des Maastrichter Vertrages. Quellen: Deutsche Bundesbank, Statistisches Bundesamt; Berechnungen des DIW Berlin; Datenund Rechenstand 7.12.2016.

Vor diesem Hintergrund verbesserte sich die öffentliche Kassenlage mehr und mehr. Seit dem Jahr 2014 schließt der öffentliche Gesamthaushalt mit einem Überschuss ab, der im vergangenen Jahr bei 0,8 Prozent in Relation zum nominalen Bruttoinlandsprodukt lag (Tabelle 1). Der tatsächliche Finanzierungssaldo ist allerdings ein unzureichender Indikator für die Beurteilung der Finanzlage, denn er spiegelt auch konjunkturelle Entwicklungen wider. Mehr Aussagekraft kommt dem sogenannten strukturellen, also dem, um konjunkturelle Einflüsse bereinigten, Finanzierungssaldo zu. Eine strukturelle Neuverschuldung von Null soll gewährleisten, dass der Staatshaushalt über einen Konjunkturzyklus ausge-

niedriger als im Jahr 2005. In den Jahren 2017 und 2018 dürften sie zwar etwas zunehmen, denn immer mehr Asylbewerber stehen nach Abschluss ihres Verfahrens dem Arbeitsmarkt zur Verfügung, danach dürften die Ausgaben für Lohnersatzleistungen aber wieder rückläufig sein.

2 Während die Zinsausgaben des Staates im Jahr 2007 noch bei 67 Milliarden Euro lagen, wurden im vergangenen Jahr 23 Milliarden Euro weniger dafür verwendet. 
glichen ist oder mit anderen Worten, die Einnahmen des Staates dauerhaft genügen, seine Ausgaben zu decken.

Der strukturelle Finanzierungssaldo des Staates ist seit dem Jahr 2013 positiv und signalisiert damit haushaltspolitischen Spielraum. Dieser wird allerdings deutlich überzeichnet. Bei der Berechnung des strukturellen Saldos gemäß des bei der deutschen Schuldenbremse zu Grunde gelegten Verfahrens, werden zwar für bestimmte Sonderfaktoren Bereinigungen vorgenommen; die aufgrund des sehr niedrigen Zinsniveaus geringeren Ausgaben werden jedoch als strukturelle Verbesserung interpretiert. Dabei kann mit dieser Entwicklung keine dauerhafte Entlastung der Staatsfinanzen verbunden sein. Sobald die Verunsicherung nachlässt, werden die zu bietenden Renditen anziehen. Erholt sich die Wirtschaft im Euroraum weiter, dürfte auch das allgemeine Zinsniveau allmählich steigen. Zwar werden zunehmende Renditen die öffentlichen Haushalte nur nach und nach im Zuge der Refinanzierung auslaufender Titel belasten, langfristig werden die Zinsausgaben aber wieder merklich zulegen. Einen Anhaltspunkt dafür, wie stark die strukturelle Verbesserung der öffentlichen Haushalte durch den Finanzierungssaldo überzeichnet wird, liefert der Vergleich zu der Entwicklung des strukturellen Primärsaldos, also des Saldos nach Bereinigung um Zinsausgaben. Während sich der strukturelle Finanzierungssaldo in Relation zum nominalen Bruttoinlandsprodukt im Jahr 2016 gegenüber dem Jahr 2008 um zwei Prozentpunkte verbesserte, ist der strukturelle Primärsaldo um lediglich einen halben Prozentpunkt gestiegen.

\section{Ausblick: Öffentliche Finanzen entwickeln sich auf den ersten Blick weiterhin günstig}

Eine Projektion der Entwicklung der öffentlichen Finanzen bis zum Jahr 2025 lässt auch für die kommenden Jahre eine eher günstige Entwicklung erwarten (van Deuverden, 2016). ${ }^{3}$ Der öffentliche Gesamthaushalt dürfte entweder ausgeglichen

3 Die Projektion basiert auf der Konjunkturprognose des DIW Berlin vom Dezember 2016 sowie der zugehörigen Mittelfristprojektion bis zum Jahr 2025 (Fichtner et. al. 2016). Aufgrund von Datenverarbeitungsfehlern bei der Erfassung sozialversicherungspflichtiger Beschäftigter dürfte diese Prognose die Entwicklung in den Jahren 2017 und 2018 zu negativ einschätzen und die öffentlichen Haushalte dürften sich etwas besser entwickelt. Die Aussagen über die längere Frist behalten jedoch Gültigkeit. Für die Finanzpolitik ist dabei unterstellt, dass das geltende Recht bis zum Ende des Projektionszeitraums fortbesteht (sogenannte „status quo“-Annahme) und Maßnahmen, die sich in der kurzen Frist abzeichnen oder hinreichend konkretisiert sind, umgesetzt werden. Dieses Annahmeset bedeutet, dass Änderungen, die sich aus gesetzlichen Regelungen und Vorschriften ergeben unterstellt werden (vgl. van Deuverden, 2016, S. 1196). 
sein oder mit einem Überschuss abschließen (Tabelle 2). Zudem wird sich der Schuldenstand gemessen am nominalen Bruttoinlandsprodukt kräftig zurückbilden - bis auf gut 48 Prozent im Jahr 2025. Diese Zahlen zeichnen allerdings ein zu positives Bild.

Tabelle 2: Wichtige finanzwirtschaftliche Kennziffern in den Jahren 2016 bis $2025^{1}$

- in Relation zum nominalen Bruttoinlandsprodukt -

\begin{tabular}{|c|c|c|c|c|c|c|c|c|c|c|}
\hline & 2016 & 2017 & 2018 & 2019 & 2020 & 2021 & 2022 & 2023 & 2024 & 2025 \\
\hline $\begin{array}{l}\text { Nominaler } \\
\text { Finanzierungssaldo }\end{array}$ & $3 / 4$ & 0 & 0 & 0 & 0 & 0 & $1 / 4$ & $1 / 2$ & $1 / 2$ & $3 / 4$ \\
\hline $\begin{array}{l}\text { Struktureller } \\
\text { Finanzierungssaldo }{ }^{2}\end{array}$ & $1 / 2$ & 0 & $-1 / 4$ & -0 & 0 & 0 & $1 / 4$ & $1 / 2$ & $1 / 2$ & $3 / 4$ \\
\hline $\begin{array}{l}\text { Primärsaldo } \\
\text { in Relation }\end{array}$ & $2^{1 / 4}$ & $11 / 2$ & $11 / 4$ & $11 / 2$ & $11 / 4$ & $11 / 4$ & $11 / 2$ & $11 / 2$ & $13 / 4$ & $13 / 4$ \\
\hline $\begin{array}{l}\text { Struktureller } \\
\text { Primärsaldo }\end{array}$ & 2 & $11 / 2$ & 1 & $11 / 4$ & $11 / 4$ & $11 / 4$ & $11 / 2$ & $11 / 2$ & $13 / 4$ & $13 / 4$ \\
\hline $\begin{array}{l}\text { Schuldenstands- } \\
\text { quote }^{3}\end{array}$ & $68^{1 / 2}$ & 66 & 64 & $61^{1 / 2}$ & $591 / 4$ & 57 & $543 / 4$ & $52^{1 / 2}$ & $501 / 2$ & $481 / 4$ \\
\hline
\end{tabular}

${ }^{1}$ In Abgrenzung der Volkswirtschaftlichen Gesamtrechnungen. ${ }^{2}$ Berechnet nach dem für die deutsche Schuldenbremse geltenden Verfahren. ${ }^{3}$ In Abgrenzung des Maastrichter Vertrages. Quellen: Deutsche Bundesbank, Statistisches Bundesamt; Berechnungen des DIW Berlin; Datenund Rechenstand 7.12.2016.

Der strukturelle Finanzierungssaldo des Staates insgesamt wird im laufenden Jahr deutlich zurückgehen und nur noch leicht positiv sein. In den kommenden beiden Jahren zeichnet sich ein strukturelles Defizit ab. Danach dürfte der Saldo in etwa ausgeglichen und ab dem Jahr 2022 wieder leicht positiv sein. Selbst im Jahr 2025 wird der strukturelle Finanzierungssaldo dabei aufgrund der Sonderentwicklung bei denZinsausgaben in den vergangenen Jahren noch unterzeichnet sein - obgleich in der Projektion ein allmähliches Anziehen der für deutsche Staatsanleihen zu bietenden Renditen unterstellt ist. Für dauerhafte Maßnahmen besteht somit insgesamt nur wenig haushaltspolitischer Spielraum.

Dies gilt umso mehr, wenn die Risiken für die Projektion berücksichtigt werden, denn diese dürften überwiegend mit Haushaltsbelastungen einhergehen. So liegt der Projektion die Annahme zugrunde, dass die wirtschaftliche Entwicklung weiterhin von einem, wenn auch etwas weniger dynamischen, Beschäftigungsaufbau begleitet wird und nach wie vor binnenwirtschaftlich getrieben ist. Zudem besteht das Risiko, dass Zinsen schneller steigen als unterstellt. 
Die Projektion beruht zudem auf der Annahme, dass der heutige Rechtsstand beibehalten wird. Dies bedeutet beispielsweise, dass die verfassungsrechtlich gebotene Anhebung von Grund- und Kinderfreibetrag zur steuerlichen Freistellung des Existenzminimums berücksichtigt wurde, eine gleichzeitige Anhebung des Kindergeldes aber nicht unterstellt worden ist; letztere ist gesetzlich nicht vorgeschrieben. Politisch wäre dies wohl kaum umsetzbar. Zudem kann die Anhebung der Freibeträge Mehreinnahmen aufgrund der kalten Progression nur mindern, nicht verhindern; steuerliche Eingriffe sind von daher wahrscheinlich.

Ferner ist unterstellt, dass trotz des Brexits der deutsche Finanzierungsbeitrag an die Europäische Union nicht wesentlich steigt und das Griechenland ab dem Jahr 2022 seinen Zinsverpflichtungen nachkommt.

Vor allem aber nehmen die Beitragssätze zu den Sozialversicherungen im Projektionszeitraum zu. Die gesetzlichen Krankenkassen müssen nach geltendem Recht ihren Zusatzbeitrag anheben, wenn ihre Haushalte aus dem Ruder zu laufen drohen. Bei der gesetzlichen Rentenversicherung muss der Beitragssatz steigen, wenn die Nachhaltigkeitsreserve am Ende des kommenden Jahres voraussichtlich das 0,3fache einer Monatsausgabe unterschreitet. In der Projektion ergibt sich ein kräftiger Anstieg der Beitragssätze um insgesamt knapp vier Prozentpunkte; dies könnte die wirtschaftliche Entwicklung stärker dämpfen als angenommen.

\section{Analyse: Der erste Blick trügt, heterogene Entwicklung einzelner Haushalte und starker Anstieg der Sozialbeiträge}

Trotz der Überschüsse ist der haushaltspolitische Spielraum für den Staat insgesamt daher begrenzt. Die gesamtwirtschaftliche Perspektive überdeckt zudem die dahinter stehende heterogene Entwicklung: Überschüssen bei den Gebietskörperschaften werden in den kommenden Jahren immer wieder Defizite bei den Sozialversicherungen gegenüber stehen.

Die Finanzlage der Gebietskörperschaften hat sich in den vergangenen Jahren deutlich aufgehellt. Sprudelnde Steuereinnahmen infolge der wirtschaftlichen Entwicklung führten dazu, dass die Einnahmen von Bund, Ländern und Kommunen kräftig stiegen. Der Bund wurde zudem auf der Ausgabenseite entlastet, denn die Ausgaben für Arbeitslosengeld gingen merklich zurück. Wegen der günstigen Entwicklung des Arbeitsmarktes fiel außerdem der allgemeine Zuschuss an die Rentenversicherung niedriger aus. Ferner konnte er am stärksten von dem niedrigen Zinsniveau profitieren. Zum einen entfällt der größte Teil der Staatsverschuldung auf ihn, zum anderen sind seine Finanzierungsbedingungen 
in der Regel günstiger als die der anderen Gebietskörperschaften (vgl. Fichtner et. al., 2012, S. 38). Der Bund wird von der weiterhin guten wirtschaftlichen Entwicklung profitieren und die Zinsausgaben dürften vorerst nur wenig zunehmen. Auch wenn für den Bundeshaushalt im Jahr 2018 zurzeit noch eine globale Minderausgabe von knapp 5 Milliarden eingeplant ist, werden auf ihn in der mittleren Frist wohl keine Finanzierungsengpässe zukommen.

Die Finanzlage der Länder hat sich in den vergangenen Jahren im Zuge der wirtschaftlichen Entwicklung ebenfalls verbessert. Dabei konnten auch die Länder von den geringen Zinskosten profitieren. Einige Länder haben ihre Haushalte zudem merklich konsolidiert. Immer mehr Länder konnten mit Überschüssen abschließen; im Jahr 2016 wiesen lediglich zwei Länder ein Haushaltsdefizit auf. Dies kann aber nicht darüber hinweg täuschen, dass viele Länder bisher zu wenig getan haben und nach wie vor nicht gut aufgestellt sind. Die Haushaltslage der Länder war im vergangenen Jahr zum großen Teil Reflex von Finanztransfers des Bundes, die dieser in Zusammenhang mit dem Zustrom von Geflüchteten im Jahr 2015 zugestanden hatte. Im laufenden wie auch im kommenden Jahr sind nochmals Mittel zugesagt, allerdings in geringerem Umfang. Die Länder insgesamt werden wohl weiter mit Überschüssen abschließen, diese werden aber geringer sein und einzelne Länder haben nach wie vor erhebliche Probleme.

Auch die kommunale Ebene erwirtschaftet seit einigen Jahren Überschüsse. Dies liegt auch daran, dass der Bund die Finanzausstattung der Kommunen in den vergangenen Jahren immer wieder verbessert hat und sich dies auch weiterhin abzeichnet. Insgesamt werden die Kommunen daher auch weiterhin Überschüsse erzielen. Allerdings ist die Finanzlage der Gemeinen nochmals heterogener als die der Länder; regionale Disparitäten haben sich in den vergangenen Jahren verfestigt und dies dürfte sich fortsetzen.

Bei den Sozialversicherungen war die Finanzlage in den vergangenen Jahren ebenfalls recht entspannt und einige konnten spürbare Überschüsse erzielen. Bei allen Sozialversicherungen legten die Einnahmen dynamisch zu, denn der Beschäftigungsaufbau fand größtenteils im sozialversicherungspflichtigen Bereich statt. Dies dürfte auch in den kommenden Jahren so bleiben, wobei der Beschäftigungsaufbau gegen Ende des Projektionszeitraums nach und nach an Dynamik verlieren wird. Dies liegt auch daran, dass - obgleich in der Projektion von einer relativ hohen Zuwanderung ausgegangen wird - die Zahl der Personen im erwerbsfähigen Alter allmählich zurückgeht.

Die Ausgabenentwicklung war bei den Sozialversicherungen hingegen recht uneinheitlich. Bei der Bundesagentur für Arbeit sanken die Ausgaben, die Arbeitslosigkeit bildete sich immer weiter zurück. Die Arbeitslosigkeit dürfte auch in den kommenden Jahren niedrig liegen und die Bundesagentur wird weiterhin mit Überschüssen abschließen. 
Bei der Rentenversicherung wurden die Ausgaben in den vergangenen Jahren dadurch gedämpft, dass die Rentenzugänge niedrig waren. Dies lag daran, dass die Alterskohorten der in den Nachkriegsjahren geborenen Versicherten, die nun das gesetzliche Renteneintrittsalter erreichten, zahlenmäßig nur schwach besetzt waren. Mehrfach konnte der Beitragssatz gesenkt werden. Die Leistungsausweitungen zu Beginn der Legislaturperiode - die Ausweitung der Mütterrente und die Einführung der „Rente ab 63“ - stoppten diese Entwicklung allerdings; ohne diese Maßnahmen läge der Beitragssatz um knapp einen Prozentpunkt niedriger.

Derzeit sind die Rentenzugänge bereits höher und werden nach und nach, wenn die geburtenstarken Jahrgänge das gesetzliche Renteneintrittsalter erreichen, weiter zunehmen. Außerdem werden die Finanzen der Rentenversicherung in den kommenden Jahren durch die beschlossene Angleichung der Ostan die Westrenten belastet - sofern hierfür nicht gleichzeitig eine Steuerfinanzierung beschlossen wird. Daher wird die Rentenversicherung in den kommenden Jahren immer wieder mit Defiziten abschließen. Für das Jahr 2022 zeichnet sich ab, dass die Schwankungsreserve ihre Untergrenze erstmalig unterschreiten dürfte. Dann wird der Beitragssatz zur gesetzlichen Rentenversicherung kräftig angehoben werden müssen. Diese Entwicklung wird sich bis weit nach Ende des Projektionszeitraums fortsetzen. Zudem werden zurzeit Maßnahmen diskutiert, die die Rentenfinanzen teils kräftig belasten würden. Werden diese beschlossen, wird der Beitragssatz noch stärker zulegen müssen als in dieser Projektion vorhergesagt.

Die Finanzlage der gesetzlichen Krankenversicherung hat sich in den vergangenen Jahren zeitweise angespannt und führte trotz guter Einnahmeentwicklung bereits zu Anhebungen der Zusatzbeiträge. Dies liegt an der hohen Ausgabendynamik bei den Krankenkassen. Neben anderen Faktoren treibt der medizinisch-technische Fortschritt die Ausgaben. Er führt nicht nur direkt zu höheren Kosten, er ermöglicht auch immer mehr Behandlungen. Außerdem schlägt sich die zunehmende Alterung in den Ausgaben nieder, denn in späteren Lebensjahren werden mehr Gesundheitsleistungen nachgefragt. Die Ausgaben je Versicherten steigen daher und werden auch weiterhin eine hohe Kostendynamik aufweisen, so dass der Zusatzbeitrag in den kommenden Jahren kräftig zunehmen wird.

Eine Projektion der mittelfristigen Entwicklung der öffentlichen Finanzen kommt zu dem Ergebnis, dass eine Anhebung der Beitragssätze bis zum Jahr 2025 um kumuliert knapp vier Prozentpunkte notwendig ist. In einem Alternativszenario, in der die Wirtschaft um durchschnittlich einen halben Prozentpunkt stärker wächst, muss er immer noch um 31/2 Prozentpunkte steigen, bei einem um einen halben Prozentpunkt geringeren Wirtschaftswachstum sogar um 41/2 Pro- 
zentpunkte. Im Basisszenario hat diese Entwicklung zur Folge, dass die Einnahmen aus Sozialbeiträgen gemessen am nominalen Bruttoinlandsprodukt im Jahr 2025 um rund zwei Prozentpunkte höher liegen wird als derzeit, während die Steuerquote aufgrund der unterstellten Anhebung der Freibeträge in etwa konstant bleibt.

\section{Begrenzte fiskalpolitische Spielräume nutzen um Wachstumsaussichten zu verbessern}

Die Projektion zur Entwicklung der öffentlichen Haushalte zeigt, dass die Lage insgesamt zwar gut ist, dass die Spielräume aber nicht groß sind, dass sie zu einem gewissen Teil nur temporär bestehen und dass sie sich bei den einzelnen Ebenen deutlich voneinander unterscheiden. Was sollte die Finanzpolitik in dieser Situation tun?

Generell sollte Finanzpolitik nachhaltig sein, d. h. sie sollte zum einen dauerhaft aufrechterhalten werden können und zum anderen darauf gerichtet sein, die Chancen für künftiges Wachstum zu erhöhen. Für Deutschland kommen empirische Studien regelmäßig zu dem Ergebnis, dass das niedrige Investitionsniveau in den vergangenen Jahren die potentielle Wirtschaftsentwicklung belastet (hat) und mahnen eine Stärkung der Investitionstätigkeit an (vgl. Expertenkommission „Stärkung von Investitionen in Deutschland“, 2015).

Auch bei den öffentlichen Investitionen lag das Investitionsniveau in den vergangenen Jahren niedrig. Hauptinvestor der öffentlichen Hand bei Bauten sind die Kommunen. Diese haben auf der Einnahmenseite relativ wenig Gestaltungsmöglichkeiten und andere Ausgaben sind meist durch gesetzliche, teilweise bundeseinheitliche Regelungen mehr oder weniger vorgegeben (vgl. Gornig et. al., 2015, S. 2024 ff.). Bei knappen Kassen wurden daher häufig die Investitionsausgaben eingeschränkt. Insbesondere in finanzschwachen Kommunen lag das Investitionsniveau niedrig und in diesen Regionen dürfte sich ein entsprechender Investitionstau gebildet haben. Da Investitionen keine dauerhaften Ausgaben begründen, könnten temporäre Spielräume dafür genutzt werden, diesen nach und nach abzubauen.

In naher Zukunft wird die demografische Entwicklung das Potentialwachstum zudem immer mehr belasten. Ohne Eingriffe werden die Beitragssätze, wie die Projektion zeigt, bis zum Jahr 2025 um vier Prozentpunkte angehoben werden müssen. Der auf Anhebungen des Zusatzbeitrags zurückgehende Teil wird dabei allein, die auf den Beitragssatz zur Rentenversicherung zurückgehende Belastung immerhin zur Hälfte von den Arbeitnehmern zu tragen sein. Trotz - angesichts der sich weiter 
anspannenden Situation auf dem Arbeitsmarkt - kräftig steigender Bruttolöhne hat dies zur Folge, dass die Nettolöhne gegen Ende des Projektionszeitraums in nominaler Rechnung nur noch um 11/2 Prozent pro Jahr zunehmen - in realer Rechnung also etwa stagnieren. Der Anreiz, Arbeit aufzunehmen dürfte durch diese Entwicklung deutlich gemindert werden - und dies in einer Situation, in der das Erwerbspersonenpotential zu schrumpfen beginnt. Dieser Prozess hat dabei im Projektionszeitraum gerade erst begonnen. Es werden weitere zehn Jahre folgen, in denen geburtenstarke Jahrgänge das gesetzliche Renteneintrittsalter erreichen und die Zahl der Rentner einer immer geringer werdenden Zahl von Personen im erwerbsfähigen Alter gegenübersteht - mit entsprechenden Anhebungen der Beitragssätze.

Vor diesem Hintergrund scheint es prioritär, die Höhe der Beitragssätze zu reduzieren. Eine Möglichkeit dazu wäre, versicherungsfremde Leistungen über Steuern zu finanzieren. Dies sind Leistungen, die nicht durch Beitragszahlungen veranlasst sind, sondern aus anderen, gesamtgesellschaftlichen Gründen gewährt werden. Ausgaben dieser Art sollten von der Allgemeinheit - also durch Steuern und nicht vom Kreis der sozialversicherungspflichtig Beschäftigten finanziert werden. Dies nimmt nicht nur ganze Gruppen, wie Studenten, Rentner, Bezieher von Gewinneinkommen oder Beamte von der Finanzierung aus, es erhöht die Lohnnebenkosten und reduziert das Arbeitsangebot.

Versicherungsfremde Ausgaben spielen vor allem bei der Krankenversicherung und bei der Rentenversicherung - wo sie durch die Ausweitung der Mütterrente und die „Rente ab 63“ zu Beginn der Legislaturperiode nochmals kräftig erhöht worden sind - eine größere Rolle und werden bisher nur unzureichend durch die gewährten Bundeszuschüsse gedeckt (vgl. van Deuverden, 2015). Die vorhandenen (dauerhaften) Haushaltsspielräume können dafür genutzt werden, diese Zuschüsse zu erhöhen. Für eine vollständige Finanzierung der versicherungsfremden Leistungen über Steuern werden sie aber nicht ausreichen. Anstatt über Steuersenkungen oder Ausgabekürzungen zu diskutieren, sollte die Schaffung weiterer Spielräume, mit denen eine Senkung der Beitragssätze finanziert werden kann, im Fokus stehen.

\section{Fazit}

Die Entwicklung der öffentlichen Haushalte hat in den vergangenen Jahren immer wieder positiv überrascht. Die Überschüsse haben nunmehr eine Debatte entfacht, wie sie zu verteilen sind. Übersehen wird dabei häufig, dass ein Teil dieser Überschüsse auf Sonderfaktoren zurückgeht und nicht von Dauer ist. Dieser Teil steht lediglich für temporäre Maßnahmen zur Verfügung und kann genutzt werden, den 
Investitionsstau abzubauen und auf diese Weise die Bedingungen für zukünftiges Wirtschaftswachstum zu verbessern. Übersehen wird häufig aber auch, dass die einzelnen Haushalte sich recht heterogen entwickeln und zudem in naher Zukunft durch die demografische Entwicklung in unterschiedlichem Ausmaß gefordert werden. Die Projektion der Finanzentwicklung in der mittleren Frist zeichnet zwar ein recht positives Bild der Staatsfinanzen insgesamt, dieses ist aber vor allem der Tatsache geschuldet, dass einige Sozialversicherungen ihre Beitragssätze kräftig anheben müssen. Dies dämpft die Bereitschaft Arbeit aufzunehmen und ist vor allem vor dem Hintergrund der sich abzeichnenden schrumpfenden Zahl von Personen im erwerbsfähigen Alter besorgniserregend. Der Teil der Überschüsse, die dauerhafter Natur sind, sollte dazu genutzt werden, die Höhe der Beitragssätze zu reduzieren, in dem mehr versicherungsfremde Leistungen durch Steuern finanziert werden.

\section{Literatur}

Expertenkommission „Stärkung von Investitionen in Deutschland“ (2015), Bericht im Auftrag des Bundesministers für Wirtschaft und Energie.

Fichtner, Ferdinand, Karl Brenke, Marius Clemens, Simon Junker, Claus Michelsen, Maximilian Podstawski, Thore Schlaak, Kristina van Deuverden (2016), „Deutsche Wirtschaft setzt Aufschwung fort - trotz schwächeren Beschäftigungsaufbaus“, DIW Wochenbericht, Jg. 83, Nr. 50, S. 1177-1188.

Fichtner, Ferdinand, Simon Junker, Guido Baldi, Jacek Bednarz, Kerstin Bernoth, Franziska Bremus, Karl Brenke, Christian Dreger, Hella Engerer, Christoph Große Steffen, Hendrik Hagedorn, Axel Heuer, Dorothee Ihle, Moritz Lerzer, Katharina Pijnenburg, Kristina van Deuverden (2012), „Krise im Euroraum bremst auch die deutsche Wirtschaft“, DIW Wochenbericht, Jg. 79, Nr. 40, S. 3-43.

Gornig, Martin, Claus Michelsen, Kristina van Deuverden, „Kommunale Infrastruktur fährt auf Verschleiß“, DIW Wochenbericht, Jg. 82, Nr. 43, S. 1023-1030.

van Deuverden, Kristina (2016), „Öffentliche Finanzen bis 2025: Nur auf den ersten Blick günstig“, DIW Wochenbericht, Jg. 83, Nr. 50, S. 1193-1202.

van Deuverden, Kristina (2015), „Finanzpolitik: Der richtige Mix von Steuern und Sozialabgaben hat Priorität!“, DIW Wochenbericht, Jg. 82, Nr. 26, S. 608-617. 